\title{
Laryngoplastik zur Medialisierung bei Lähmungen und Defekten der Glottis
}

\section{Medialisation Laryngoplasty to Treat Vocal Fold Paralysis and Glottal Defects}

Dirk Mürbe, Tadeus Nawka

Laryngoplastiken zur Medialisierung der Stimmlippe haben sich als effektive operative Verfahren zur Stimmverbesserung bei einseitigen Stimmlippenlähmungen und Substanzdefekten der Glottis etabliert. Umfangreiche Erfahrungen liegen insbesondere zur Medialisierungsthyroplastik vor. Über ein Fenster im Schildknorpel wird das Stimmlippengewebe durch Einsetzen von körpereigenem Material (Knorpel) oder externen Implantaten (Titan, Gore-Tex etc.) medialisiert. Bei ausgeprägter Lateralstellung einer gelähmten Stimmlippe ist die Kombination der Medialisierungsthyroplastik mit einer Arytenoidadduktion erfolgversprechend, um auch den Spalt im knorpeligen Teil der Glottis zu verkleinern. Die Indikationsstellung zur Laryngoplastik darf nicht ausschließlich auf den bildmorphologischen Befund des Kehlkopfes reduziert werden, sondern erfordert eine modularisierte Stimmdiagnostik. Abhängig vom Befund ist ein therapeutisches Stufenkonzept zu beachten. Bei geringer glottaler Schlussinsuffizienz sind zunächst eine initiale Stimmübungsbehandlung und ggf. eine Injektionsglottoplastik vor der externen Laryngoplastik zu erwägen.

LERNZIEL

In diesem Beitrag werden Verfahren der Medialisierungsthyroplastik als operativer Ansatz bei Stimmlippenlähmungen und Substanzdefekten der Glottis vorgestellt. Unter Beachtung eines adäquaten diagnostischen Instrumentariums werden typische Indikationen für eine Laryngoplastik dargestellt, die das stimmtherapeutische Oeuvre von Stimmübungsbehandlung und Injektionsglottoplastik vervollständigt. Der Überblick operativer Techniken schließt auch die Arytenoidadduktion ein, welche als Erweiterung der Medialisierungsthyroplastik bessere funktionelle Ergebnisse insbesondere bei lähmungsbedingten großen Defiziten des Stimmlippenschlusses in Aussicht stellt.

\section{Einführung}

Unter dem Begriff Laryngoplastik wird die Larynxskelettchirurgie (Laryngeal Framework Surgery) mit externem Zugang im Bereich des äußeren Hals verstanden. In der Praxis sind das meist die chirurgischen Verfahren zur Optimierung der Stimmlippenposition bei einseitiger Stimmlippenlähmung. Die dazu vorwiegend angewandte Operationsmethode, die Medialisierungsthyroplastik, ist der Gruppe der Approximationslaryngoplastiken zuzuordnen. Die gelähmte Stimmlippe wird vom äußeren Larynxskelett her medialisiert. Ziel ist die Wiederherstellung des Stimmlippenschlusses bzw. die weitgehende Verringerung der glottalen Schlussinsuffizienz. Zudem erhöht sich bei diesem Vorgehen die Stimmlippenspannung, und die Stimmlippenkontur kann geglättet werden, damit die Stimmlippen im membranösen Anteil besser schließen. Dies trifft für weitere, neben der einseitigen Stimmlippenlähmung bestehende Indikationen zu, wie Substanzdefekte der Glottis nach Tumorresektionen oder die Atrophie des Musculus vocalis bei Stimmstörungen im höheren Lebensalter. 
Geschichtlich geht die Thyroplastik zur Medialisierung der Stimmlippe auf einen von Payr im Jahr 1915 veröffentlichten Vorschlag zurück, nach dem ein verbesserter Stimmlippenschluss durch ein vom Schildknorpelblatt imprimiertes Knorpelstück erreicht werden kann [1]. Im weiteren Verlauf wurden zahlreiche Modifikationen der Thyroplastik beschrieben, die sich vorwiegend durch die verwendeten Materialien zur Medialisierung unterscheiden. In einer von Isshiki veröffentlichten Systematik der Larynxskelettchirurgie wird die Medialisierungslaryngoplastik mit Knorpel als Thyroplastik Typ 1 bezeichnet [2]. Bei Isshiki findet sich auch der Vorschlag zur Arytenoidadduktion [3]. Durch Rotation des Aryknorpels wird der Stimmlippenschluss im hinteren, kartilaginären Glottisbereich verbessert. Dieses Verfahren ergänzt den Medialisierungseffekt der Thyroplastik.

Auf weitere Typen der Laryngoplastik, wie die Extensions- und Relaxationslaryngoplastik wird in diesem Beitrag nicht eingegangen.

Abzugrenzen von den Laryngoplastiken der Larynxskelettchirurgie ist die Injektionsglottoplastik oder Stimmlippenaugmentation als Therapiemethode bei geringen glottalen Schlussinsuffizienzen, die in einem weiteren Beitrag in diesem Heft beschrieben ist.

\section{Diagnostik}

Die Indikationsstellung zur Medialisierungsthyroplastik mit oder ohne Arytenoidadduktion muss stets auf den Befunden einer multimodalen Stimmdiagnostik basieren, welche den Empfehlungen der Europäischen Laryngologischen Gesellschaft (ELS) folgt [4]. Die verschiedenen Module der Stimmdiagnostik schließen die umfassende perzeptive Beurteilung des Stimmklanges, die visuelle Beurteilung der Stimmlippenschwingung mittels Videostrobolaryngoskopie bzw. Hochgeschwindigkeitsglottografie, die apparative Stimmanalyse und Stimmleistungsdiagnostik, die Erfassung ausgewählter aerodynamischer Maße sowie die standardisierte Selbstbeurteilung der eigenen Stimme ein.

Die infolge einseitiger Stimmlippenlähmung oder Tumorresektion bestehenden glottalen Schlussinsuffizienzen und Schwingungsirregularitäten führen sowohl zu rauen als auch behauchten Komponenten des Stimmklangs. In der videostrobolaryngoskopischen Beurteilung sind insbesondere das Ausmaß der Schlussinsuffizienz im Verlauf der membranösen Stimmlippe und die Position des Aryknorpels zu beurteilen. Die apparative Stimmschallanalyse objektiviert die perzeptiv beschriebenen Veränderungen des Stimmklanges, während das funktionelle Defizit in der Stimmleistungsbeurteilung mittels Stimmfeldmessung quantitativ und qualitativ darstellbar ist. Bei einseitigen Stimm- lippenlähmungen kommt der Beurteilung der maximalen Phonationsdauer besonderer Stellenwert zu. Nicht zuletzt ermöglicht die systematische Selbstauskunft des Patienten durch Fragebögen (z. B. VHI) eine Relativierung der klinischen Untersuchungsbefunde zur individuellen Reflexion der Stimmstörung und zur kommunikativen Teilhabe.

\section{Indikationen}

Die Medialisierungsthyroplastik kommt insbesondere bei Stimmstörungen aufgrund einer einseitigen Stimmlippenlähmung zum Einsatz, die mit dem Stufenschema von Stimmübungstherapie und Injektionsglottoplastik nicht zufriedenstellend behandelt werden können. Des Weiteren wird das Verfahren in der rekonstruktiven Phonochirurgie nach Entfernung von Stimmlippentumoren genutzt. Dabei liegt die operative Intention nicht in einer Medialisierung der gesamten membranösen Stimmlippe, sondern im gezielten Ausgleich glottaler Defekte, um wieder eine glatte Kontur in der Ebene der tumorchirurgisch behandelten Stimmlippe zu erreichen. Zudem ist eine Medialisierungsthyroplastik, gegebenenfalls auch beidseits, bei ausgeprägter Atrophie des Musculus vocalis bei Presbyphonie zu erwägen, wenn durch das eingangs genannte nicht bzw. gering invasive Stufenschema keine ausreichende Stimmverbesserung zu erzielen ist.

Bei intermediärer oder lateraler Position der gelähmten Stimmlippe und inadäquater Stimmlippenspannung ist eine Größe des Glottisspaltes $>3 \mathrm{~mm}$ als ein stimmdiagnostisches Teilkriterium für eine Präferenz der Medialisierungsthyroplastik im Vergleich zur Injektionsglottoplastik einzuschätzen [5]. Neben dem Ausmaß der zu überbrückenden Spaltbildung sind Narbenbildungen im Bereich der Glottis bei der Auswahl der operativen Strategie zu beachten. Grenzen der Injektionsglottoplastik ergeben sich zudem bei einseitig überzogener Massenbelastung der Stimmlippe durch das injizierte Material, woraus trotz gutem Glottisschluss ein irreguläres Schwingungsverhalten resultiert.

Bei lähmungsbedingt sehr großer glottaler Schlussinsuffizienz, insbesondere im hinteren Bereich der Glottis, und bei deutlicher Verkippung des Aryknorpels mit Niveaudifferenz der Stimmlippen kann auch mit einer Medialisierungsthyroplastik kein hinreichender Stimmlippenkontakt bei Phonation erzielt werden. Dann sollte der Eingriff um eine Arytenoidadduktion erweitert werden, wobei durch die operativ realisierte Rotation des Aryknorpels der Funktionsausfall des Musculus cricoarytenoideus lateralis kompensiert werden soll und eine über die Knorpelimprimierung hinausgehende Medialisierung der Stimmlippe erreicht wird [3]. 

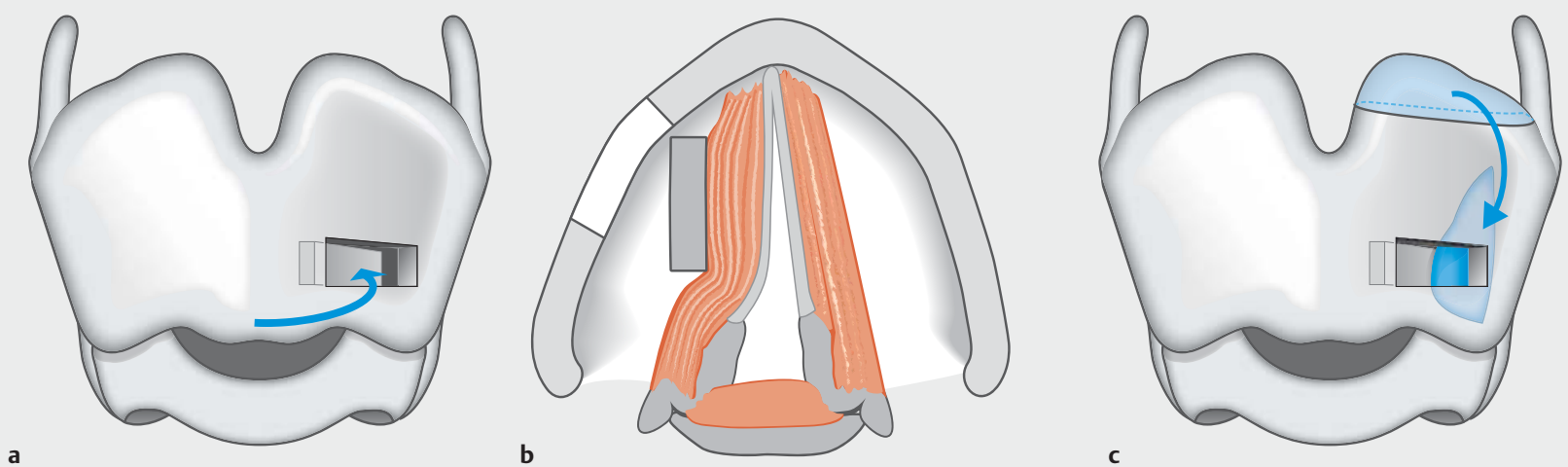

- Abb. 1 Schematische Darstellung der zentralen operativen Schritte einer Medialisierungsthyroplastik mit körpereigenem Knorpel. a Anlage eines Knorpelfensters im unteren Schildknorpelbereich in durch Landmarken und Abstandsbeziehungen definierter Position. b Imprimierung des durch Anlage des Fensters entstandenen Knorpelstückes entsprechend dem Ausmaß der Lateralstellung der gelähmten Stimmlippe bzw. der Position und Größe des Glottisdefektes. c Fixierung des imprimierten Knorpels durch einen zuvor am oberen Schildknorpelrand entnommenen schmalen Knorpelstreifen, der das Knorpelfenster von innen verriegelt.

Für den Zeitverlauf der Indikationsstellung für eine Medialisierungsthyroplastik muss neben den individuellen Befunden die Prognose des Störungsbildes einbezogen werden, um das grundsätzliche Vorgehen des stimmtherapeutischen Stufenkonzepts im Einzelfall auch hinsichtlich einer Primärindikation für eine Laryngoplastik abzuändern, bspw. bei irreversibler Läsion des Nervus vagus mit Lateralstellung der gelähmten Stimmlippe. Andererseits kann bei unsicherer Prognose die Injektionsglottoplastik die Zeit bis zur definitiven Laryngoplastik überbrücken. Das übergeordnete Ziel ist die zeitnahe Wiederherstellung oder Verbesserung der Stimmfunktion.

\section{Chirurgische Techniken}

Die chirurgischen Techniken der Medialisierungsthyroplastik schließen verschiedene Varianten der ursprünglich vorgeschlagenen Impression und Fixierung eines rechteckigen Knorpelstückes im unteren Schildknorpelbereich ein. Alternativ zum körpereigenen Knorpel stehen heute Implantate aus verschiedenen biokompatiblen Materialien zur Verfügung, die bspw. als GoreTex-Streifen, in Form eines Silikonkeiles oder als Titanspange eingesetzt werden [6]. Alle Techniken haben eine optimale und sichere Positionierung des Implantates mit präziser Medialisierung und Remodellierung der Stimmlippe zum Ziel. Nachfolgend wird zusammenfassend die von den Autoren in vielen klinischen Konstellationen bevorzugte Technik der transzervikalen Stimmlippenmedialisierung mit körpereigenem Knorpel dargestellt.
Intraoperativ wird nach horizontalem Hautschnitt in einer Hautfalte kurz oberhalb der Schildknorpelunterkante der Musculus sternohyoideus auseinandergedrängt und die laterale Schildknorpelplatte (Lamina thyroidea) dargestellt. Unter konsequenter Beachtung der Landmarken und definierten Abstandsbeziehungen wird im unteren Schildknorpelbereich mit Skalpell oder Bohrer ein Knorpelfenster angelegt, wobei das endolaryngeale Perichondrium zirkulär mobilisiert, aber intakt belassen wird. Nach Feinmodellierung des passager entnommenen Knorpelstückes wird dieses durch das Fenster wiedereingesetzt und entsprechend dem Ausmaß und der Lokalisation des Glottisdefektes in der Tiefe positioniert. Die Fixierung des imprimierten Knorpels erfolgt durch einen weiteren, zuvor am oberen Schildknorpelrand entnommenen schmalen Knorpelstreifen, der durch 90-Grad-Drehung das Knorpelfenster von innen verriegelt ( $\triangleright$ Abb. 1).

Bei Erweiterung der Medialisierungsthyroplastik um eine Arytenoidadduktion wird an der Hinterkante des Schildknorpels ein halbkreisförmiges Knorpelstück entfernt und der Processus muscularis des Aryknorpels dargestellt. Durch Öffnung des Gelenkes und Lösung der Bänderverbindungen wird der Aryknorpel mobilisiert und schließlich mit einer Naht durch den Processus muscularis angeschlungen, sodass der Faden durch das Fenster des Schildknorpels nach vorn gezogen werden kann. Daraus resultiert eine Innenrotation des Aryknorpels mit Einwärtsbewegung der gelähmten Stimmlippe ( $\triangleright$ Abb. 2). 


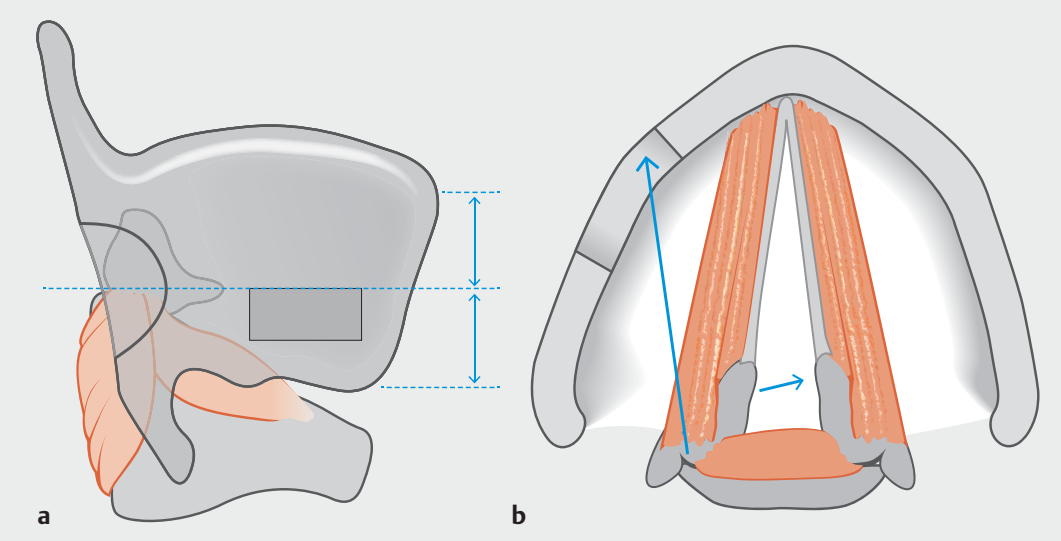

- Abb.2 Schematische Darstellung der Erweiterung einer Medialisierungsthyroplastik um eine Arytenoidadduktion. a Darstellung des Processus muscularis des Aryknorpels nach Anlage eines weiteren, halbkreisförmigen Knorpelfensters im dorsalen Bereich des Schildknorpelblattes. b Innenrotation des Aryknorpels mit Einwärtsbewegung der gelähmten Stimmlippe durch Fadenzug am Processus muscularis nach vorn.
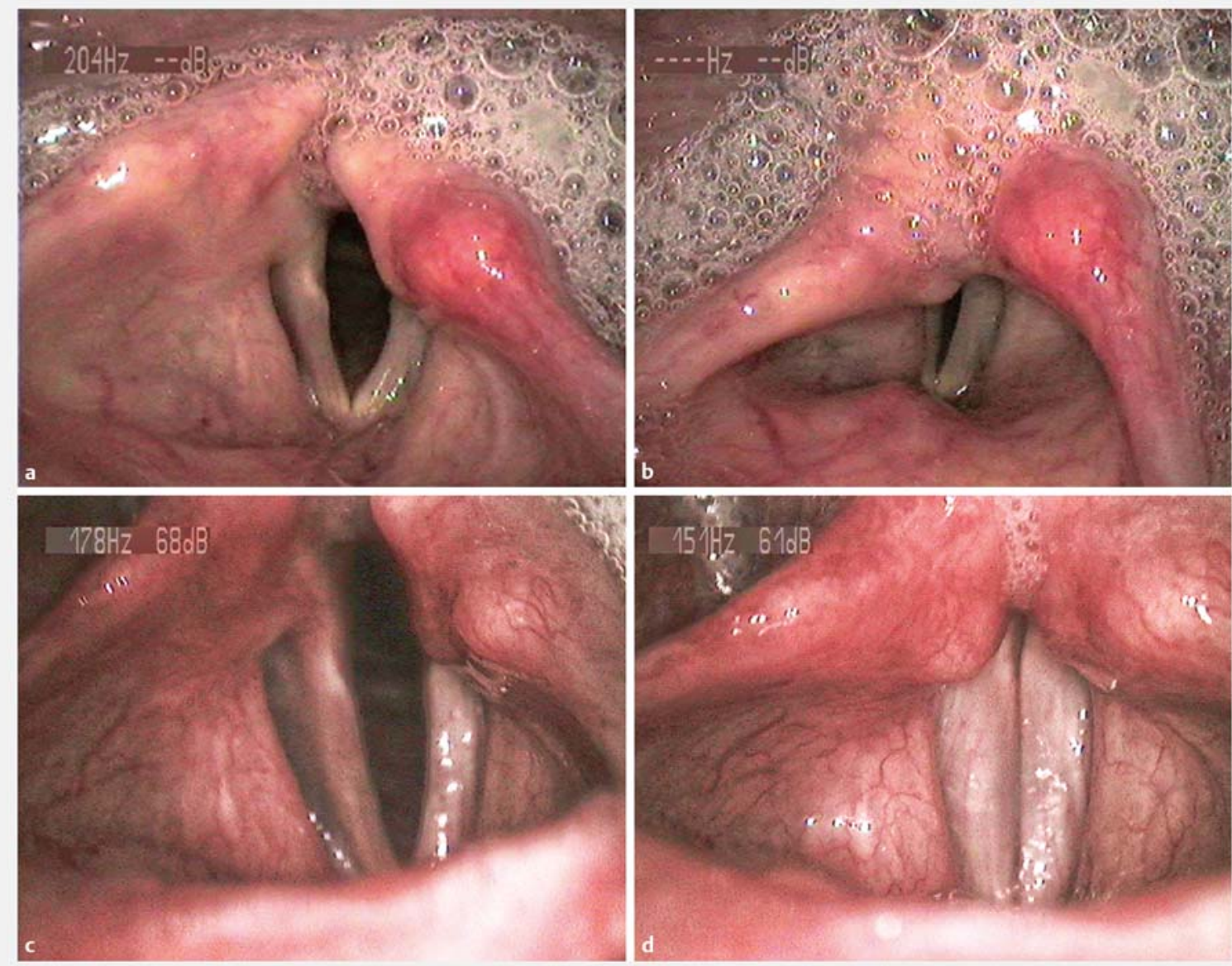

- Abb. 3 Laryngostroboskopische Befunde bei Stimmlippenlähmung links infolge einer Parese des Nervus vagus links. a präoperativ mit Lateralstellung und Exkavation der gelähmten Stimmlippe in Respirationsstellung und b präoperativ mit deutlicher Schlussinsuffizienz in Phonationsstellung, c postoperativ nach Medialisierungsthyroplastik mit Arytenoidadduktion links mit permanenter Medialisierung der gelähmten linken Stimmlippe in Respirationsstellung und $\mathbf{d}$ postoperativ mit dichtem Stimmlippenschluss in Phoniationsstellung. 

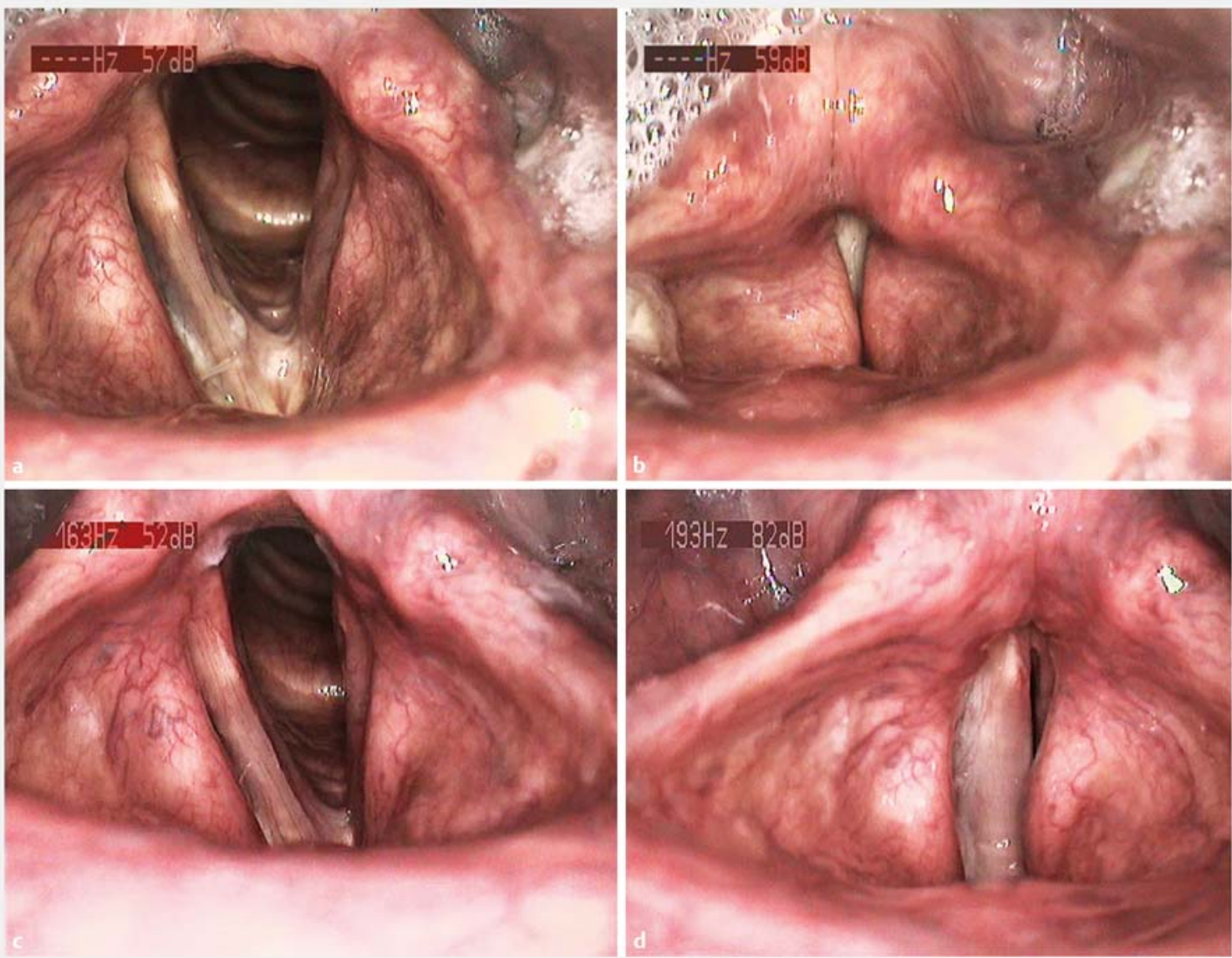

Abb. 4 Laryngostroboskopische Befunde bei Z.n. partieller Chordektomie links aufgrund eines T1-Stimmlippenkarzinoms a mit deutlichem Substanzdefekt und unregelmäßigem Rand der linken Stimmlippe in Respirationsstellung und $\mathbf{b}$ bei Ersatzphonation mit Taschenfalteneinsatz, c postoperativ nach Medialisierungsthyroplastik links mit permanenter Medialisierung und Konturbegradigung der linken Stimmlippe in Respirationsstellung, d postoperativ mit Restitution glottischer Phonation mit verbessertem Stimmlippenschluss.

Die operativen Techniken der Stimmlippenmedialisierung werden sowohl in Lokalanästhesie als auch Vollnarkose durchgeführt. Operationen in Lokalanästhesie erlauben die unmittelbare perzeptive Kontrolle des Ergebnisses der Medialisierung intraoperativ, sind allerdings in Abhängigkeit der Compliance des Patienten und weiterer Rahmenbedingungen nicht immer umsetzbar. Auch in Vollnarkose ist intraoperativ, über eine Larynxmaske flexibel endoskopisch, die Kontrolle des Medialisierungseffektes der Stimmlippen möglich [7]. Ein operatives Vorgehen in Vollnarkose ist insbesondere bei Arytenoidadduktion aufgrund der ausgedehnteren Exploration des Schildknorpels und des Aryknorpels zu favorisieren.

\section{Ergebnisse}

Ergänzend zu Stimmübungsbehandlungen und Injektionsglottoplastiken hat sich die Medialisierungsthyroplastik als verlässliches phonochirurgisches Modul im stimmtherapeutischen Stufenkonzept bei einseitiger Stimmlippenlähmung oder glottischen Substanzdefekten etabliert. Die mit einer Medialisierungsthyroplastik verbundenen Erwartungen liegen in der Wiederherstellung oder Verbesserung des Stimmlippenschlusses und Optimierung biomechanischer Rahmenbedingungen für eine effiziente glottische Stimmschallproduktion. Auf Basis einer multimodalen Stimmdiagnostik nach dem ELS-Protokoll erscheinen einige stimmdiagnostische Parameter besonders geeignet, die Versorgungsqualität nach einer Medialisierungsthyroplastik zu beurteilen. Diese umfassen insbesondere maximale Phonationsdauer, subjektive und objektive Heiserkeitsbewertung, videolaryngostroboskopische Beurteilung, Stimmleistungsprofil und subjektive Selbsteinschätzung. 
Auch wenn dem Anspruch einer multimodalen stimmdiagnostischen Bewertung der funktionellen Ergebnisse nach Medialisierungsthyroplastik in den einzelnen Studien nicht vollumfänglich entsprochen werden kann, ist der Versorgungserfolg anhand von ausgewählten Stimmparametern vielfach belegt. Dabei sind bspw. eine Verbesserung der subjektiven Selbstbewertung der Stimme, eine Verlängerung der maximalen Phonationsdauer, eine Verbesserung der Stimmleistung mit größerem Stimmumfang, eine Verringerung der Glottisschlussinsuffizienz sowie eine Verbesserung des Stimmklanges mit geringerer Heiserkeit im Ergebnis einer Medialisierungsthyroplastik beschrieben [812].

Auch für die Erweiterung der Medialisierungsthyroplastik mit einer Arytenoidadduktion bei sehr großer Schlussinsuffizienz im hinteren Bereich der Glottis liegen umfangreiche Untersuchungen vor. Diese belegen funktionelle Verbesserungen der Stimmfunktion mit signifikanter Steigerung von maximaler Phonationsdauer und Stimmumfang $[13,14]$. Anhand videolaryngoskopischer Beurteilung des Stimmlippenschlusses fanden Li et al. [15] keine Unterschiede im Vergleich von Medialisierungsthyroplastik mit und ohne Arytenoidadduktion, wohingegen Chang et al. [16] Vorteile für die Erweiterung mit einer Arytenoidadduktion beschreiben. ( Abb. 3, \Abb.4).

FAZIT

Laryngoplastiken stellen einen unverzichtbaren Bestandteil der operativen stimmtherapeutischen Möglichkeiten dar. Bei glottalen Substanzdefekten bzw. Schlussinsuffizienzen ist die Medialisierungsthyroplastik eine etablierte Option, wenn konservative Therapie oder Injektionslaryngoplastik nicht ausreichen. Bei einer großen Schlussinsuffizienz sollte die Medialisierungsthyroplastik mit einer Arytenoidadduktion verbunden werden. In diesen Fällen sind stabile Verbesserungen der Stimmfunktion für verschiedene Parameter nachgewiesen.
Schlüsselwörter

Laryngoplastik, Thyroplastik, Arytenoidadduktion, Stimmlippenlähmung, Heiserkeit, Dysphonie

Interessenkonflikt

Die Autoren geben an, dass kein Interessenkonflikt besteht.

\section{Autorinnen/Autoren}

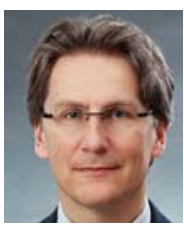

\section{Dirk Mürbe}

Prof. Dr. med., Medizinstudium an den Universitäten Rostock, Dublin und Dresden. Parallel zum Medizinstudium Gesangsstudium an der Hochschule für Musik Carl Maria von Weber Dresden, Abschluss mit künstlerischem Diplom. Facharzt für Phoniatrie und Pädaudiologie sowie Facharzt für HNO-Heilkunde. 2010 Professor für Phoniatrie und Pädaudiologie an der TU Dresden mit Leitung der Abteilung Phoniatrie und Audiologie sowie des Sächsischen Cochlear Implant Centrum Dresden. 2018 Berufung an die Charité Berlin und Bestellung zum Direktor der Klinik für Audiologie und Phoniatrie. Klinisch-wissenschaftliche Schwerpunkte in den Bereichen Stimmstörungen und Phonochirurgie sowie $\mathrm{Cl}$-Versorgung und Hör-/Spracherwerb. Wissenschaftliche Tätigkeit und Lehraufgaben am Studio für Stimmforschung der Hochschule für Musik Dresden und an der Universität Mozarteum Salzburg.

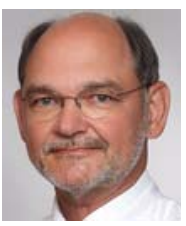

\section{Tadeus Nawka}

Univ.-Prof. Dr. med., seit 2009 in der Charité - Universitätsmedizin Berlin/Klinik für Audiologie und Phoniatrie tätig, Leiter des Stimmzentrums der Charité in Berlin. Von 1999-2009 Professor für Phoniatrie und Pädaudiologie an der Universität Greifswald und seit 2017 Präsident der European Academy of Phoniatrics.

Institut

Klinik für Audiologie und Phoniatrie, Charité

Universitätsmedizin Berlin

\section{Korrespondenzadresse}

Prof. Dr. med. Dirk Mürbe

Direktor der Klinik für Audiologie und Phoniatrie

Charité Universitätsmedizin Berlin

Augustenburger Platz 1

13353 Berlin

E-Mail: dirk.muerbe@charite.de 


\section{Literatur}

[1] Payr E. Plastik am Schildknorpel zur Behebung der Folgen einseitiger Stimmbandlähmung. Dtsch Med Wochenschr 1915; 43: $1265-1270$

[2] Isshiki N, Morita H, Okamura H et al. Thyroplasty as a new phonosurgical technique. Acta Otolaryngol 1974; 78: 451 457

[3] Isshiki N, Tanabe M, Sawada M. Arytenoid adduction for unilateral vocal cord paralysis. Arch Otolaryngol 1978; 104: $555-558$

[4] Dejonckere PH, Bradley P, Clemente P et al. A basic protocol for functional assessment of voice pathology, especially for investigating the efficacy of (phonosurgical) treatments and evaluating new assessment techniques. Guideline elaborated by the Committee on Phoniatrics of the European Laryngological Society (ELS). Eur Arch Otorhinolaryngol 2001; 258: $77-82$

[5] Rosen CA, Simpson B Hrsg. Operative Techniques in Laryngology. Springer; 2008: 978-3-540-25806-3

[6] Crolley VE, Gibbins N. One hundred years of external approach medialisation thyroplasty. Journal of Laryngology \& Otology 2017; 131: $202-208$

[7] Kanazawa T, Watanabe Y, Hara M et al. Arytenoid adduction combined with medialization laryngoplasty under general anesthesia using a laryngeal mask airway. Am J Otolaryngol 2012; 33: 303-307

[8] Overton L, Adams K, Shah RN et al. Longitudinal voice outcomes after type I Gore-Tex thyroplasty for nonparalytic glottic incompetence. Ann Otol Rhinol Laryngol 2017; 126 : $14-19$

[9] Bertelsen C, Reder L. Efficacy of type I thyroplasty after endoscopic cordectomy for early-stage glottic cancer: Literature review. Laryngoscope 2018; 128: 690-696
[10] Desuter G, Dedry M, Schaar B et al. Voice outcome indicators for unilateral vocal fold paralysis surgery: a review of the literature. Eur Arch Otorhinolaryngol 2018; 275: 459 468

[11] Dominguez L, Villarreal R, Simpson CB. Voice Outcomes of Lipoinjection Versus Medialization Laryngoplasty for Nonparalytic Glottic Insufficiency. Laryngoscope 2018: doi:10.1002/lary.27573

[12] McLaughlin CW, Swendseid B, Courey MS et al. Long-term outcomes in unilateral vocal fold paralysis patients. Laryngoscope 2018; 128 : $430-436$

[13] Tokashiki R, Hiramatsu H, Shinada E et al. Analysis of pitch range after arytenoid adduction by fenestration approach combined with type I thyroplasty for unilateral vocal fold paralysis. J Voice 2012; 26: $792-796$

[14] Konomi U, Watanabe Y, Komazawa D. Sex Differences in Pitch Range and Speech Fundamental Frequency After Arytenoid Adduction and Thyroplasty. J Voice 2016; 30: 362 370

[15] Li A], Johns MM, Jackson-Menaldi C et al. Glottic closure patterns: type I thyroplasty versus type I thyroplasty with arytenoid adduction. J Voice 2011; 25: 259-264

[16] Chang J, Schneider SL, Curtis 」 et al. Outcomes of medialization laryngoplasty with and without arytenoid adduction. Laryngoscope 2017; 127: $2591-2595$

\section{Bibliografie}

DOI https://doi.org/10.1055/a-0682-3030

Sprache · Stimme · Gehör 2018; 42: 192-198 (c) Georg Thieme Verlag KG Stuttgart · New York ISSN 0342-0477 